Rhinitis, Sinusitis, Otitis

\title{
Naturheilkunde in der Erkältungssaison
}

\author{
Zur Prävention wie auch zur Symptomlinderung von Erkältungskrank- \\ heiten ist so manches "Kraut" gewachsen. Prof. A.-M. Beer stellt die \\ wichtigsten naturheilkundlichen Strategien gegen Rhinitis \& Co. vor, \\ die Sie an Ihre Patienten weitergeben können.
}

\begin{abstract}
? Welche naturheilkundlichen Maßnahmen bieten sich bei der Mittelohrentzündung an?

Die Möglichkeiten der klassischen Naturheilverfahren bei der Mittelohrentzündung liegen vor allem in der Prävention. Bei einer bestehenden Otitis media sind sie eher begrenzt.

Der Belüftungsverbesserung dienen Inhalationen mit Kamillenblütenkonzentrat (z. B. Kamillosan). Kochsalz-Nasenspülungen können einer Ausweitung des Infektes vorbeugen. Alternativ kann ein entzündungshemmendes Nasenspray (z.B. Rinupret ${ }^{\circ}$ Pflege) empfohlen werden. Hier ist neben Meersalz auch
\end{abstract}

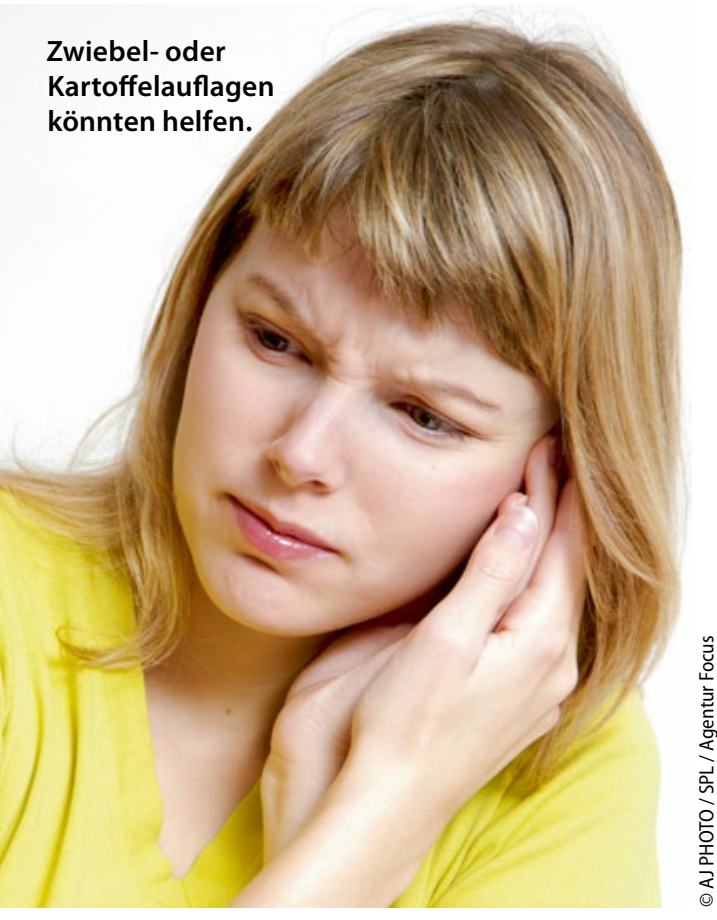

Eukalyptusöl und Aloe vera enthalten. Begleitend kann zur Infektabwehr eine Pflanzenkombination aus Kapuzinerkresse und Meerrettichwurzel (Angocin ${ }^{\circ}$ Anti-Infekt N) verordnet werden.

Zur Schmerzbekämpfung bieten sich Zwiebel- oder Kartoffelauflagen auf das Mastoid an. Zu Beginn einer Otitis media kann phytotherapeutisch ein Sonnenhutpräparat $\left(\right.$ Echinacin $\left.^{\circledR}\right)$ gegeben werden. Eine Mikrowellenbestrahlung des Mastoids kann durch immunstimulierende Prozesse die Heilung beschleunigen. Neuraltherapeutisch können Injektionen an das Mastoid mit Procain $1-2 \%$ durchgeführt werden.

\section{Frage: Welche Maßnahmen helfen bei Rhinitis und Sinusitis?}

$\mathrm{Zu}$ den Fertigpräparaten Sinupret extract ${ }^{\oplus}$ und Umckaloabo ${ }^{\circ}$ liegen ausreichend klinische Studien vor. Bei der Nasenreflextherapie nach Röder werden mit einem Nasenreflexöl die Reflexzonen der drei Nasengänge behandelt. Darüber erreicht man eine Hypersekretion, Schleimhautabschwellung und einen gesteigerten Lymphabfluss.

Bei beginnender Rhinitis kann ein Senfmehlfußbad eingesetzt werden:

- Fußwanne bis zu zwei Drittel mit $37^{\circ} \mathrm{C}$ warmem Wasser füllen, 2 EL Senfmehl dazugeben, Füße eintauchen.

- Heißes Wasser nachgießen, damit über 20 min. eine konstante Wassertemperatur besteht.

- Danach Füße mit lauwarmen Wasser abspülen, anschließend gut abtrocknen. Füße mit Johanniskrautöl (z.B. Rotöl ${ }^{\circ}$ ) oder einem anderen

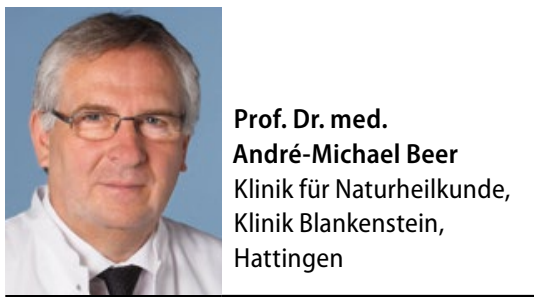

guten Pflanzenöl (Olivenöl) einreiben, Wollsocken anziehen.

- Nachruhe 30 Minuten oder Schlafengehen.

Das Bad ruft eine Hautrötung hervor, manchmal tritt diese Rötung erst nach mehreren Bädern auf. Sollte die Rötung am nächsten Tag noch vorhanden sein, einen Tag mit der Behandlung aussetzen.

Bei Kindern werden $1 / 2$ EL Senfmehl (3-6 Jahre) bzw. 1 EL Senfmehl (6-17 Jahre) verwendet.

\section{Ausstellung Naturheilkunde von einst bis jetzt}

Vom 15. April bis 31. Juli 2016 findet in der Klinik Blankenstein, Hattingen, eine Ausstellung zum Thema "Naturheilkunde von einst bis jetzt" statt. Die klassischen Naturheilverfahren bilden den Ursprung unserer europäischen Medizin. Durch das Engagement der Pioniere der Naturheilkunde sind diese Verfahren auch heute noch ein wichtiger Bestandteil der Medizin und geraten zunehmend ins Blickfeld sowohl der Schulmedizin als auch der Öffentlichkeit. Die Wegbereiter - von Hahn, Kneipp über Bilz bis Lahmann und Schweninger - werden in einer Zusammenstellung gewürdigt, die es in dieser Form noch nie gegeben hat. Diese Pioniere haben die Naturheilkunde über mehr als 250 Jahre hinweg bereichert und weiterentwickelt. Gezeigt werden Exponate aus mehr als zehn nationalen und internationalen Sammlungen, wie etwa seltene Buchexemplare und Originalabbildungen. Aber auch Originalfilme und -Tonaufnahmen erwarten die Besucher.

Gruppenführungen werden angeboten.

(Tel. 02324-396485) 